\title{
BMJ Open Family Violence Curricula in Europe (FAVICUE): a cross-sectional descriptive study protocol
}

\author{
Raquel Gómez Bravo, ${ }^{1}$ Charilaos Lygidakis, ${ }^{1}$ Gene Feder, ${ }^{2}$ Robert A P Reuter, ${ }^{3}$ \\ Claus Vögele ${ }^{1}$
}

To cite: Gómez Bravo R, Lygidakis C, Feder G, et al. Family Violence Curricula in Europe (FAVICUE): a cross-sectional descriptive study protocol. BMJ Open 2019;9:e024519. doi:10.1136/ bmjopen-2018-024519

- Prepublication history and additional material for this paper are available online. To view these files, please visit the journal online (http://dx.doi. org/10.1136/bmjopen-2018024519).

Received 30 May 2018

Revised 20 December 2018

Accepted 27 December 2018

Check for updates

(C) Author(s) (or their employer(s)) 2019. Re-use permitted under CC BY-NC. No commercial re-use. See rights and permissions. Published by BMJ.

${ }^{1}$ Research Unit INSIDE (Integrative Research Unit on Social and Individual Development), Institute for Health and Behaviour, University of Luxembourg, Esch-surAlzette, Luxembourg ${ }^{2}$ School of Social and Community Medicine, Centre for Academic Primary Care, University of Bristol, Bristol, UK ${ }^{3}$ Research Unit ECCS (Education, Culture, Cognition and Society), Institute of Applied Educational Sciences, University of Luxembourg, Esch-sur-Alzette, Luxembourg

Correspondence to Dr Raquel Gómez Bravo; raquel.gomezbravo@uni.lu

\section{ABSTRACT}

Introduction Family violence (FV) is a widespread public health problem of epidemic proportions and serious consequences. Doctors may be the first or only point of contact for victims who may be hesitant or unable to seek other sources of assistance, and they tend not to disclose abuse to doctors if not specifically asked. A comprehensive healthcare response is key to a coordinated communitywide approach to FV, but most of the practising physicians have received either no or insufficient education or training in any aspect of FV. Training of medical students concerning FV is often delivered in an inconsistent or ad hoc manner. The main aim of this project, Family Violence Curricula in Europe (FAVICUE), is to (1) describe current FV education delivery in European medical universities (undergraduate period) and during the specialist training in general practice (GP)/family medicine (FM) (postgraduate residency programme), and (2) compare it with WHO recommendations for FV curriculum.

Methods and analysis This is the protocol of a crosssectional descriptive study consisting of two self-report online surveys (for undergraduate and postgraduate training, respectively) with 40 questions each. For both surveys, general practitioners, residents, medical students and professionals involved in their education from countries of the European region will be identified through the European Regional Branch of the World Organization of National Colleges, Academies and Academic Associations of General Practitioners/Family Physicians (WONCA Europe) and will be invited to provide information regarding the training on FV. Descriptive tests will be carried out and a thematic analysis will be conducted on the open-ended questions.

Ethics and dissemination Ethics approval has been obtained by the University of Luxembourg (ERP 17-015 FAVICUE). The results will provide important information concerning current curricula on FV, and can be used for mapping the educational needs and planning the implementation of future training interventions. They will be published and disseminated through WONCA Europe and its networks.

\section{INTRODUCTION}

Family violence (FV) is a widespread public health problem of epidemic proportions and serious consequences. ${ }^{1}$ In addition to FV constituting a violation of human rights, ${ }^{2}$ its

\section{Strengths and limitations of this study}

To the best of our knowledge, this will be the first study carried out in the European region to describe family violence curricula during undergraduate and postgraduate specialist training in general practice/ family medicine.

- This study adopts a cross-sectional descriptive design consisting of two self-report online surveys with approximately 40 questions each: the length of the survey may decrease response rate and lead to a bias.

- The surveys are divided into 14 sections exploring a wide range of domains of the curricula.

- The questionnaires will address different types of family violence separately: intimate partner violence, child abuse and neglect, female genital mutilation and elder abuse, among others.

- The study will be carried out through a scientific society providing a unique access to its members, who are located across the countries of the European region.

consequences involve serious damage to the physical, mental and social well-being of individuals and families. FV, also named domestic violence, domestic abuse or battering, includes intimate partner violence (IPV), child abuse/neglect and elder abuse, among others.

Doctors may be the first or only point of contact for victims of FV who may be hesitant or unable to seek other sources of assistance. ${ }^{34}$ Although statistics show that abused women use healthcare services more than non-abused women, and they also identify healthcare providers as the professionals they would most trust with disclosure of abuse, ${ }^{5}$ they tend not to disclose abuse to doctors if not specifically asked. ${ }^{6}$ Healthcare professionals are in a unique position to offer a safe and confidential environment not only to facilitate disclosure of violence, but also to offer appropriate support and referral to other resources and services. ${ }^{2}$ 
A comprehensive healthcare response is key to a coordinated community-wide approach to FV, but most of the practising physicians have received either no or insufficient education or training in any aspect of $\mathrm{FV}^{78}$ Prior research has found that training of medical students concerning $\mathrm{FV}$ is often delivered in an inconsistent or ad hoc manner, ${ }^{78}$ and healthcare professionals report feeling inadequately trained to care for victims of abuse. ${ }^{9}$ In order to assume their roles and responsibility, it is necessary to sensitise them towards FV and provide them with the information and tools necessary to respond sensitively and effectively. ${ }^{2}$ Training in FV is likely to improve clinical practice in such cases. ${ }^{10}$ WHO and the National Institute for Health and Care Excellence have published guidelines for health services responding to IPV, emphasising the urgent need to improve the education of frontline healthcare professionals. ${ }^{11}$

The Family Violence Curricula in Europe (FAVICUE) study aims at describing current FV curricula delivery both in European undergraduate medical programmes and in the specialty training in general practice (GP)/family medicine (FM) (postgraduate residency programmes), and compares them with the corresponding WHO recommendations regarding IPV, sexual violence (SV), child abuse (CA) and elder abuse (EA) training to include them in the healthcare curriculum.

The 2013 WHO guidelines strongly recommend training for healthcare providers at a prequalification level and to healthcare providers in-service for IPV and $\mathrm{SV}$, considering them as best practices. ${ }^{1}$

A structured, integrated CA training in the curricula for all medical students and health professionals in training was already recommended by the WHO 2002 world report on violence and health. ${ }^{12}$ Early detection of child maltreatment and early intervention can help to minimise the likelihood of further violence and the longterm health and social consequences. In order to increase the capacity of frontline professionals, a call for special training is made including the minimum knowledge that this should cover. ${ }^{13}$

Evidence-based education on EA for all primary healthcare workers was suggested based on the findings from the WHO 2008 multicountry study in elder abuse and neglect. ${ }^{14}$ This recommendation has been made considering that EA rates are predicted to increase as many countries are experiencing rapidly ageing populations and the health sector needs to be trained to appropriately detect, respond and treat this increasing health concern. $^{15}$

\section{METHODS AND ANALYSIS \\ Design}

This study adopts a cross-sectional descriptive design consisting of two self-report online surveys (one for postgraduate ('FAVICUE I') and one for undergraduate training ('FAVICUE II')) with approximately 40 questions each (see online supplementary appendix 1 and 2), with open-ended and close-ended questions, divided into 14 different sections, as presented in table 1 .

These sections will explore different domains (see table 1) of the curricula dividing them into subsections according to the different types of $\mathrm{FV}$, also named domestic violence, domestic abuse or battering, that includes: IPV, child abuse and neglect, female genital mutilation and EA, among others, which will be addressed separately in the survey.

It is important to remark that IPV refers to behaviour by an intimate partner or ex-partner who causes physical, sexual or psychological harm, including physical aggression, sexual coercion, psychological abuse and controlling behaviour. It is mentioned that it is one of the most common forms of violence against women, but this does not exclude any other gender-based violence that results in, or is likely to result in, physical, sexual or mental harm or suffering to women, including threats of such acts, coercion or arbitrary deprivation of liberty, whether occurring in public or in private life. ${ }^{16}$

The items were chosen in line with those previously used by Alpert et al, ${ }^{17}$ Valpied and colleagues, ${ }^{18}$ Srivastava and Coles $^{19}$ and Potter and Feder, ${ }^{20}$ and taking into account WHO recommendations, ${ }^{12-15}$ aiming for a comprehensive assessment of all aspects of FV curricula.

\section{Patient and public involvement}

Neither patients nor the public were involved in the design of this study. To ensure wide dissemination of the outcomes, they will be presented in scientific meetings worldwide and publications will be sought in peer-reviewed open-access journals.

\section{Participants}

\section{Inclusion criteria}

The target population of participants are general practitioners (GPs), including those who are in training, working in primary care, in rural, urban or emergency settings and those involved in training. For both surveys, GPs and residents from countries of the European region will be contacted to participate in the survey through the email list and social media accounts of the European Regional Branch of the World Organization of National Colleges, Academies and Academic Associations of General Practitioners/Family Physicians (WONCA Europe). WONCA Europe is an academic and scientific society, with 47 member organisations and represents more than 120000 family physicians in Europe.

For the purposes of this study, the European Region is defined according to the WHO Regional Office for Europe,$^{21}$ comprising an extensive geographical area of 53 countries between the Atlantic and the Pacific oceans; WONCA Europe also conforms to this definition. The survey will be conducted with the support of three of the WONCA Europe networks, and one special interest group of the world umbrella organisation (WONCA):

1. The WONCA Special Interest Group on Family Violence (WONCA SIGFV); 
Table 1 The sections of the two FAVICUE surveys

\section{FAVICUE I}

1 Profile

2 Training setting

3 Personal background: GP trainee or senior GP, years of practice after the GP vocational training.

4 Types of FV:

- If there is no training on FV: plans to introduce it in the curriculum and reasons why it is not currently included.

- Training on FV: compulsive or elective activity and teaching methods used.

5 Content of the training:

- Topics covered: epidemiology, risk factors, physical and mental health consequences, child protection, gender issues, FGM, honoured-based violence, identification, management, community services and case studies.

- Who delivers the teaching, year during which it is offered, total number of hours.

- How and when to ask about each type of violence.

6 Monitoring

\section{FAVICUE II}

Profile

Medical school

Training on FV at the University

Types of FV:

- If there is no training on FV: plans to introduce it in the curriculum and reasons why it is not currently included.

- Training on FV: compulsive or elective activity and teaching methods used.

Format of the training:

- Who delivers the teaching.

- Year during which it is offered.

- Total number of hours.

\section{Documentation}

8 Confidentiality

9 Safety: How to assess safety.

10 Referrals: How to refer patients who disclose abuse or when there is a suspicion of abuse.

11 Protocol: Is there any kind of national or local protocol for dealing with each type of violence?

12 Policy: Is there a national policy about each type of violence?

13 Personal opinion about the current quantity of teaching provided and whether or not there should be a formal teaching on FV in the curriculum.

14 Future plans to develop/change the teaching provision.
Content of the training:

- Does the training have specific learning objectives?

- Does the training have specific learning outcomes, topics covered?

- Topics covered: epidemiology, risk factors, physical and mental health consequences, child protection, gender issues, FGM, honoured-based violence, identification, management, community services and case studies.

- How and when to ask about each type of violence.

Documentation

Confidentiality

Safety: How to assess safety.

Referrals: How to refer patients who disclose abuse or when there is a suspicion of abuse.

\section{Monitoring}

Practice:

- Does the teaching involve opportunities to apply this knowledge?

- Is it embedded as part of a clinical rotation or attachment?

- Barriers and facilitators

Personal opinion about the current quantity of teaching provided and whether or not there should be a formal teaching on FV in the curriculum.

Future plans to develop/change the teaching provision.

FAVICUE, Family Violence Curricula in Europe; FGM, female genital mutilation; FV, family violence; GP, general practice.

2. The Vasco da Gama Movement (a network of WONCA Europe) for trainees and junior family doctors, with a representative member for each European country;

3. The European Academy of Teachers in General Practice/Family Medicine (a network of WONCA Europe);

4. The working group on Mental Health and Family Violence of the European Network for Prevention and Health Promotion in Family Medicine and General Practice (a network of WONCA Europe).

\section{Exclusion criteria}

Participants who are neither GPs nor medical students or those professionals who are not involved in their training.

\section{Recruitment and informed consent}

Physicians and medical students will receive an online invitation to participate in the study including the link to the platform where they will find all the information related to the project, its objectives and expected 
outcomes. It will be distributed through the emailing list of the aforementioned networks. Each one of them have country representatives, who can provide information and insights about the standards of the curriculum. They can participate or forward the email to other potential participants who have knowledge of FV training in specialty training programmes.

Moreover, programme leads and coordinators of the curricula (eg, deans, faculty or programme directors, professors, course coordinators, lecturers, researchers, educators) of European Medical Schools will receive the survey, as well as students' associations.

The first page of the online survey contains the consent form and information on how the data will be treated. Volunteers have to agree to these terms and conditions by clicking the respective button before being taken to the next pages of the online survey.

\section{Sample size}

Our sample consists of GPs, GP trainees, medical students and teaching professionals from the 41 countries represented in WONCA Europe: Andorra, Armenia, Austria, Belarus, Belgium, Bosnia and Herzegovina, Bulgaria, Croatia, Czech Republic, Denmark, Estonia, Finland, France, Germany, Greece, Hungary, Iceland, Ireland, Israel, Italy, Kazakhstan, Latvia, Lithuania, Luxembourg, Macedonia, Malta, Netherlands, Norway, Poland, Portugal, Romania, Russia, Serbia, Slovakia, Slovenia, Spain, Sweden, Switzerland, Turkey, Ukraine and the UK.

According to the world directory of Medical Schools, ${ }^{22} 23$ there are at least 452 medical schools in Europe.

Regarding the postgraduate curriculum, the last research carried out for EURACT, the European Academy of Teachers in General Practice/Family Medicine, Specialist training for GP/FM is firmly established internationally and is a prerequisite for becoming an 'Official/Licensed/Specialist' GP in all EU/EEA (European Economic Area) countries surveyed except Albania, Austria, Lithuania and Ukraine out of the 39 countries surveyed. ${ }^{24}$ We could expect to find some variations of the curricula across some countries as, for example, Spain has 17 different regions with variations of the official programme, Portugal counts on four training regions and the Netherlands has eight institutes in charge of the training - although there are national standards for the curriculum that we will explore.

\section{Data analysis}

For each survey, data will be collected during a 12-month consecutive period, starting on 16 January 2018. Quantitative analysis will be carried out using SPSS: descriptive statistics will be computed and $\chi^{2}$, t-tests and non-parametric tests and bivariate tests, where applicable, will be performed. A thematic analysis will be conducted on the open-ended questions using NVivo. ${ }^{25}$
ETHICS AND DISSEMINATION

\section{Ethical approval}

This study will be conducted according to the guidelines laid down in the Declaration of Helsinki and the guidelines of the Ethics Review Panel (ERP) of the University of Luxembourg. The study design was approved on 19 September 2017.

\section{Participant's information and consent}

Informed consent will be obtained from all participants before any data collection ensues. This will include the right to decline and to withdraw from the research once it has started. They will not receive any incentive for the participation in the study. If participants wish to be informed of the results of the project and relevant publications, they can contact the principal investigator through the contact provided in the online survey.

\section{Data protection}

The data will be collected anonymously, by default. The data used in this research project will be collected through a web-based service (LimeSurvey) hosted on a server within the network of the University of Luxembourg. The transmission of data to this server will be secured using the HTTPS protocol. There will be no link between the data and the participant. The server and the LimeSurvey application are managed by the central information technology (IT) department, which is in control of granting access to the server. Once the data collection has been completed, the data will be copied to a centrally managed file share of the UL main file server dedicated to the project. After the validation of the download, the data set on the web server will be deleted. Access to the project file share is only provided by the IT department on authorisation of the owner of the file share. To ensure the confidentiality of the data and to follow the principle of privacy by design, the data will be stored in an AES encrypted ZIP archive. The decryption password will only be known to the members of the project and not be shared with a third party. Temporary copies of the data made by accessing the ZIP archive and used for data analysis will be deleted once the analysis has been completed. The ZIP archive will be kept for 10 years in the project share drive and will be destroyed after this period in accordance with the ethics guidelines of the University Luxembourg.

\section{Dissemination plan}

Study findings will be disseminated through peer-reviewed publications, conference presentations, posters and social media channels. The research findings will provide important information concerning current curricula on FV, enhancing the knowledge by facilitating mapping the current training provision in the European region. The outcomes of the study have the potential to help in the identification of educational needs, and in planning the implementation of future training interventions or improvement of existing ones. 


\section{DISCUSSION}

Historically, most medical care providers have not been taught the skills to recognise and treat victims of FV. Even if awareness has increased among the medical profession in the last decade, we expect to find insufficient levels of training provision. Such a result would underline the significant gap between the recognition of $\mathrm{FV}$ as a public health problem of epidemic proportions and its serious consequences, and the provision of adequate training of first-line professionals to address this important problem. ${ }^{11}$ The results of this study will help to improve our understanding of the situation in the European Region, raise awareness for the importance of the contents of medical curricula and suggest further implementations.

\section{Comment}

To the best of our knowledge, this will be the first study carried out in the European region to describe Family Violence curricula during undergraduate medical school training and postgraduate specialist training in GP/FM.

\section{Limitations}

The main limitation of this study relates to the use of self-report questionnaires, with which we will collect information from GPs and medical students. This heterogeneity of the respondents and the length of the survey may lead to response bias. Voluntary participation may also influence the outcomes, as participants who are more aware and sensitive to FV are more likely to respond to the survey. According to Borgiel et al, the best recruitment results come from: a personal approach by a known physician to the potential participant; having professionals of influence in the medical field or respected peers undertaking the recruitment, and; the personal level of interest and commitment. ${ }^{26}$ Considering also that colleagues participating in scientific networks tend to be more active, the response rates may be ultimately acceptable.

Even though efforts will be made to reach a large number of respondants, our sample may not be representative, as there is no way to control how the surveys will be distributed in each country through the representative members in the networks and coordinators of that specific topic might not receive them. However, as these participants have a specific commitment within the scientific society and their own countries, and a special interest in primary care and education and/or FV, we do believe that they will be able to answer the questionnaire as accurately as possible.

Even if some participants underwent other types of formation after completing their undergraduate and/or postgraduate training, we believe their answers will reflect the actual curricula. This is because the survey concerns the teaching the actual teaching provision in Medical Universities and GP vocational training programmes and it is directed to those who are in contact with these programmes.
Despite the WHO recommendations for a FV curriculum that includes assessing communication and clinical skills, inappropriate attitudes among healthcare providers (victim blaming or expecting patient to leave relationship immediately) and self-care techniques for providers, these have not been specifically included in the questionnaire to keep the general focus on the basic training and avoid increasing the extension of the questionnaire. Even if participants always have the possibility to write down more specific points related to the training if they consider it appropriate, this broad approach might imply another bias in our study. However, as colleagues participating in scientific networks tend to be more active, their responses may be ultimately acceptable.

Discrepancies are expected to be found in our studies considering the diversity of the participants, which are consistent with what the literature describes. ${ }^{17}$ According to Alpert EJ et al, there are many reasons to explain disparities between the report of the deans related to what they are teaching and what students report they are being taught in domestic violence. Normally, students report fewer educational offers than the coordinators of the same school. The possible explanations for this finding are several, like the perception about the curriculum, the schedule of the subject, whether it is the case of a single presentation and it is not re-emphasised over time, or it is part of multiple courses, or clinical settings, etc. ${ }^{17}$ In case they are discovered, we will address the problem by analysing deeper the concrete case of this country.

Acknowledgements We are grateful for the kind support received from WONCA Europe (WONCA Europe: European Regional Branch of the World Organization of National Colleges, Academies and Academic Associations of General Practitioners/ Family Physicians) from its president, Dr Anna Stadval, and its networks, especially the Vasco da Gama Movement (the Junior Doctors Network of Family Medicine in Europe), its president, Dr Claire Marie Thomas, and the Vasco da Gama Movement Family Violence group, the WONCA Special Interest Group on Family Violence and its co-Chairs Dr Hagit Dascal-Weichhendler and Professor Dr Kelsey Hegarty, and past Chair Dr Lodewijk Pas, the European Network for Prevention and Health Promotion in Family Medicine and General Practice (EUROPREV) and its president Dr Mateja Bulc, the European Academy of Teachers in General Practice/Family Medicine (EURACT) and its president, Dr Jo Buchanan, the International Section of the Spanish Society of Family and Community Medicine (SEMFYC) and its Representative member of Family and Gender Violence, Dr M Carmen Fernández Alonso, and all the colleagues who took part in the survey, sharing their knowledge and providing information to make this research possible.

Collaborators WONCA Special Interest Group on Family Violence.

Contributors RGB serves as the principal investigator for this study. She designed the study, conducted the literature search and wrote the manuscript. RGB, CL, GF, RAPR and CV participated in the design of the study and drafting of the protocol. CL advised on statistical methods, reviewed, edited and commented on the different versions of this manuscript. GF and $\mathrm{CL}$ gave their expert opinion on medical education in the training of medical students and GP trainees and RAPR on education. CV edited the manuscript prior to submission and gave his expert opinion concerning research design. All authors have read the draft critically to make contributions and also approved the final text.

Funding RGB is employed by the University of Luxembourg as a full time PhD student. No external funds support her research.

Competing interests RGB is member of the executive of the WONCA SIGFV and CL is the honorary secretary of WONCA Europe.

Patient consent for publication Not required. 
Ethics approval Ethics approval has been obtained from the University of Luxembourg (ERP 17-015 FAVICUE).

Provenance and peer review Not commissioned; externally peer reviewed.

Open access This is an open access article distributed in accordance with the Creative Commons Attribution Non Commercial (CC BY-NC 4.0) license, which permits others to distribute, remix, adapt, build upon this work non-commercially, and license their derivative works on different terms, provided the original work is properly cited, appropriate credit is given, any changes made indicated, and the use is non-commercial. See: http://creativecommons.org/licenses/by-nc/4.0/.

\section{REFERENCES}

1. World Health Organization. Responding to intimate partner violence and sexual violence against women: WHO clinical and policy guidelines. Geneva: WHO, 2013. Accessible online at. http://apps. who.int/iris/bitstream/10665/85240/1/9789241548595_eng.pdf

2. World Health Organization. Preventing intimate partner and sexual violence against women: taking action and generating evidence. Geneva, Switz: World Health Organization, 2010. Accessible online at. http://www.who.int/violence_injury_prevention/publications/ violence/9789241564007_eng.pdf

3. Ansara DL, Hindin MJ. Formal and informal help-seeking associated with women's and men's experiences of intimate partner violence in Canada. Soc Sci Med 2010;70:1011-8.

4. Feder G, Davies RA, Baird K, et al. Identification and Referral to Improve Safety (IRIS) of women experiencing domestic violence with a primary care training and support programme: a cluster randomised controlled trial. Lancet 2011;378:1788-95.

5. Feder GS, Hutson M, Ramsay J, et al. Women exposed to intimate partner violence: expectations and experiences when they encounter health care professionals: a meta-analysis of qualitative studies. Arch Intern Med 2006;166:22-37.

6. Hegarty K, Feder G, Ramsay J. Identification of partner abuse in health care settings: Should health professionals be screening? In: Roberts G, Hegarty K, Feder G, eds. Intimate partner abuse and health professionals. London, England: Elsevier, 2006:79-92.

7. Frank E, Elon L, Saltzman LE, et al. Clinical and personal intimate partner violence training experiences of u.s. medical students. $J$ Womens Health 2006;15:1071-9.

8. Wathen CN, Tanaka M, Catallo C, et al. Are clinicians being prepared to care for abused women? A survey of health professional education in Ontario, Canada. BMC Med Educ 2009;9:Article 34.
9. Davidson LL, Grisso JA, Garcia-Moreno C, et al. Training programs for healthcare professionals in domestic violence. J Womens Health Gend Based Med 2001;10:953-69.

10. Connor PD, Nouer SS, Mackey SN, et al. Intimate partner violence education for medical students: toward a comprehensive curriculum revision. South Med J 2012;105:211-5.

11. National Institute for Health and Care Excellence. Domestic violence and abuse: how health services, social care and the organisations they work with can respond effectively. NICE Public Health Guidance 50. London: Department of Health, NICE, 2014.

12. Krug EG, Mercy JA, Dahlberg LL, et al. World Report on Violence and Health. Geneva: World Health Organisation, 2002:1083-8.

13. World Health Organisation. Preventing child maltreatment: a guide to taking action and generating evidence. 2006 http://apps.who.int/iris/ bitstream/handle/10665/43499/9241594365 eng.pdf?sequence=1

14. World Health Organisation. A Global Response to Elder Abuse and Neglect: Building Primary Health Care Capacity to Deal with the Problem Worldwide: Main Report. France. 2008 http://www.who.int/ ageing/publications/ELDER_DocAugust08.pdf

15. World Health Organisation. Elder abuse: fact sheet. http://www.who. int/en/news-room/fact-sheets/detail/elder-abuse (updated June 2018).

16. Nations U. Declaration on the elimination of violence against women. New York: UN, 1993.

17. Alpert EJ, Tonkin AE, Seeherman AM, et al. Family violence curricula in U.S. medical schools. Am J Prev Med 1998;14:273-82.

18. Valpied J, Aprico K, Clewett J, et al. Are Future Doctors Taught to Respond to Intimate Partner Violence? A Study of Australian Medical Schools. J Interpers Violence 2017;32:2419-32.

19. Srivastava M, Coles J. Mapping family violence curriculum in asian and pacific nations. honors degree of bachelor medical science: Monash University, 2015.

20. Potter LC, Feder G. Domestic violence teaching in UK medical schools: a cross-sectional study. Clin Teach 2018;15:382-6.

21. Introducing WHO/Europe. World Health Organization Regional Office for Europe. 2018. URL http://www.euro.who.int/en/about-us

22. World directory of Medical Schools. https://archive.is/ 20141023092001/http://wdoms.org/

23. 10. English-Language Medical Schools in Europe. https://www. medicaldoctor-studies.com/

24. EURACT. 11. Specialist Training Database. http://euract. woncaeurope.org/specialist-training-database/

25. Hennink M, Hutter I, Bailey A. Qualitative research methods. London: SAGE, 2011.

26. Borgiel AE, Dunn EV, Lamont CT, et al. Recruiting family physicians as participants in research. Fam Pract 1989;6:168-72. 